\title{
Estabilidad Primaria en Implantes Inmediatos Versus Implantes Colocados en Hueso Maduro: Un Estudio Clínico Retrospectivo
}

\author{
Primary Stability in Immediate Implants Versus Implants Placed \\ in Mature Bone: A Retrospective Clinical Study
}

\begin{abstract}
David Chávarri-Prado'; Aritza Brizuela-Velasco'; Pablo Ortiz de Urbina-Comerón²; Markel Diéguez-Pereira'; Esteban Pérez-Pevida ${ }^{3}$; Iratxe Viteri-Agustín ${ }^{4}$; Alejandro Estrada-Martínez ${ }^{1}$ \& Oier Montalbán-Vadillo ${ }^{3}$
\end{abstract}

CHÁVARRI-PRADO, D.; BRIZUELA-VELASCO, A.; ORTIZ DE URBINA-COMERÓN, P.; DIÉGUEZ-PEREIRA, M.; PÉREZPEVIDA, E.; VITERI-AGUSTÍN, I.; ESTRADA-MARTÍNEZ, A. \& MONTALBÁN-VADILLO, O. Estabilidad primaria en implantes inmediatos versus implantes colocados en hueso maduro: un estudio clínico retrospectivo. Int. J. Odontostomat., 14(2):230-235, 2020.

RESUMEN: La estabilidad primaria es un requisito importante para la supervivencia y éxito de los implantes durante la osteointegración. En los últimos años, los implantes inmediatos postextracción han demostrado ser una opción de tratamiento exitosa y predecible para la reposición de dientes con mal pronóstico, pero surge la duda de si dichos implantes alcanzan valores de estabilidad primaria comparables a aquellos colocados en hueso maduro. Comparar la estabilidad primaria de implantes inmediatos colocados en alveolos postextracción con la de implantes colocados en hueso maduro. Se llevó a cabo un estudio clínico retrospectivo, con los datos recogidos sobre 175 implantes, colocados en 175 pacientes. Todos los implantes colocados pertenecían al modelo Essential Cone (Klockner Implant System) y se dividieron en dos grupos: implantes inmediatos (Grupo $A, n=31$ ) e implantes colocados en hueso maduro (Grupo B, $n=144$ ). La estabilidad primaria de todos los implantes se midió mediante torque de inserción y análisis de frecuencia de resonancia con Osstell ISQ. No se encontraron diferencias estadísticamente significativas respecto a la estabilidad medida a través del torque de inserción $(26,29+10,07 \mathrm{Vs} 25,76+9,72 \mathrm{~N} / \mathrm{cm})$ pero sí que se encontraron diferencias significativas en la medida de la estabilidad primaria mediante AFR, siendo inferiores los valores correspondientes a los implantes colocados en los alveolos post exodoncia $(60,74 \pm 6,17$ en sentido VL y $62,19 \pm 7.64$ en sentido MD frente a $68,34 \pm 6.26$ en sentido VL y $69,29 \pm 7.98$ en sentido MD obtenidos en los implantes colocados en hueso maduro). El torque de inserción de los implantes inmediatos es similar al de los implantes colocados en hueso maduro, pero sus valores ISQ son significativamente inferiores, lo que demuestra un mayor grado de micromovimiento, y por consiguiente, un mayor riesgo de fracaso durante el período de osteointegración.

PALABRAS CLAVE: estabilidad implantaria, estabilidad primaria, análisis de frecuencia de resonancia, torque de inserción, implante inmediato.

\section{INTRODUCCIÓN}

La pérdida dentaria conlleva una serie de consecuencias sobre el paciente que afectan tanto a su función masticatoria, como a su estética, su fonación e incluso puede provocar repercusiones de índole psicológica. Desde el descubrimiento de la capacidad de osteointegración del titanio por el profesor Per-Ingvar
Brånemark, la implantología oral se ha convertido en el tratamiento de primera elección para este problema (Misch et al., 2005; Brånemark, 1988). En la actualidad, el tratamiento mediante la colocación de implantes dentales presenta unas altas tasas de predictibilidad, ya que alcanzan el $95 \%$ de supervi-

\footnotetext{
${ }^{1}$ Departamento de Cirugía y Especialidades médico-quirúrgicas, Facultad de Medicina y Odontología, Universidad de Oviedo, Oviedo, España.

${ }^{2}$ Práctica privada. Salamanca, España.

${ }^{3}$ Departamento de Cirugía, Facultad de Medicina, Universidad de Salamanca, Salamanca. España.

4. Departamento de Cirugía, Ginecología y Obstetricia, Facultad del Deporte y Ciencias de la Salud, Universidad de Zaragoza, Huesca, España.
} 
CHÁVARRI-PRADO, D.; BRIZUELA-VELASCO, A.; ORTIZ DE URBINA-COMERÓN, P.; DIÉGUEZ-PEREIRA, M.; PÉREZ-PEVIDA, E.; VITERI-AGUSTÍN, I.; ESTRADAMARTÍNEZ, A. \& MONTALBÁN-VADILLO, O. Estabilidad primaria en implantes inmediatos versus implantes colocados en hueso maduro: un estudio clínico retrospectivo. Int. J. Odontostomat., 14(2):230-235, 2020.

vencia a los cinco años y el $90 \%$ a los diez, cuando se colocan en hueso maduro y con protocolos de carga convencional (Pjetursson et al., 2004).

Una de las claves del éxito de un implante es la estabilidad (Misch et al.). La estabilidad de un implante se define como la ausencia de movilidad clínica bajo una carga determinada, y ha sido ampliamente reconocida como un factor clave para lograr y mantener la osteointegración (Lin et al., 2007; West \& Oates, 2007; Rowan et al., 2015). Para lograr una correcta osteointegración se necesita una buena estabilidad primaria y que el implante no se mueva, ya que se ha comprobado que en implantes que superan cierto umbral de microvimiento (150 micras) cabe esperar un predominio de la encapsulación fibrosa en detrimento de la osteointegración (Bilhan et al., 2010; Dos Santos et al., 2011; Rowan et al.; Han et al., 2016). La presencia de micromovimiento induce la reabsorción de hueso de la interfase y crea un gap que puede provocar el fracaso del implante (Bilhan et al.).

Los principales factores que influyen en la estabilidad primaria, y en consecuencia en la osteointegración, son la densidad ósea, el diseño del implante y la técnica quirúrgica empleada. (Dos Santos et al.; Gehrke \& Marin, 2015; Malchiodi et al., 2016). Desde un punto de vista clínico cobra especial relevancia el prestar atención a estos factores, para poder minimizar riesgos durante el período de osteointegración.

Dada la importancia de la estabilidad implantaria en el éxito o fracaso del implante, surge la necesidad de medir dicha estabilidad con la finalidad de monitorizar el proceso de osteointegración. A día de hoy, existen multitud de métodos capaces de medir la estabilidad, pero entre ellos destacan el torque de inserción y el Análisis de Frecuencia de Resonancia (AFR). El torque se define como la resistencia que un implante experimenta cuando se somete a un avance rotacional en dirección apical alrededor de su eje. Este no puede ser excesivo ya que aumenta el estrés periimplantario del hueso generando reabsorción ósea, pérdida de osteointegración y la consecuente pérdida del implante (Brizuela-Velasco et al., 2015). El principal inconveniente del torque es que solamente se puede medir una vez, en el momento de la colocación del implante. Por lo tanto, sólo es capaz de medir la estabilidad primaria, pero no la secundaria, ya que tendríamos que recurrir al torque de desinserción para ello. En cambio, el AFR proporciona una información clínica relevante sobre el estado de la interfase hue- so-implante en cualquier etapa tras la colocación del implante (Koh et al., 2009; Bilhan et al.; Sennerby, 2015; Mccullough \& Klokkevold, 2017). El análisis de frecuencia de resonancia (AFR) es un método no invasivo que proporciona mediciones objetivas de la rigidez de la unión hueso-implante, basado en la determinación de la frecuencia natural de vibración del implante. Dicha frecuencia natural de vibración va a depender de la masa del implante, y sobre todo, de la rigidez de su unión con el hueso, de tal forma que cuanto mayor sea esta rigidez, más alta va a ser su frecuencia natural de vibración. Los dispositivos RFA nos permiten averiguar cuál es dicha frecuencia, para en base a ella, determinar su grado de estabilidad en una escala de valores ISQ (Implant Stability Quotient) comprendidos entre 0 y 100 (Santamaría-Arrieta et al., 2016).

Tras la pérdida de un diente, el protocolo tradicional empleado durante años ha consistido en esperar varios meses antes de la colocación del implante, para que se produjera la cicatrización del alveolo (Adell et al., 1981). Sin embargo, en los últimos tiempos se ha promulgado para ciertos casos la colocación del implante en el alveolo de manera inmediata a la extracción, con el objetivo de conseguir una mejor estética y reducir los tiempos de tratamiento (Lazzara, 1989).

La colocación inmediata del implante presenta ciertas ventajas importantes como la reducción de la morbilidad y del tiempo de tratamiento asociado a la reposición de un diente, ya que minimiza el número de procedimientos quirúrgicos al combinar la extracción, la colocación del implante y el injerto óseo si fuera necesario en una sola cita. Esto hace que la satisfacción del paciente tras el tratamiento sea mayor (Werbitt \& Goldberg, 1992; Lindeboom et al., 2006; Agliardi et al., 2010).

Actualmente la literatura revela que no existen diferencias significativas en la tasas de éxito y supervivencia de los implantes inmediatos colocados en alveolos frescos y en los implantes colocados en hueso maduro (Chen et al., 2004; Malchiodi et al.). convirtiéndose en una opción terapéutica perfectamente viable para su uso clínico.

Respecto a la estabilidad primaria, no hay unanimidad y existe cierta controversia ya que la mayoría de autores consideran que no hay diferencias significativas en los valores ISQ de los implantes colocados post-extracción respecto a los implantes colocados en 
CHÁVARRI-PRADO, D.; BRIZUELA-VELASCO, A.; ORTIZ DE URBINA-COMERÓN, P.; DIÉGUEZ-PEREIRA, M.; PÉREZ-PEVIDA, E.; VITERI-AGUSTÍN, I.; ESTRADAMARTÍNEZ, A. \& MONTALBÁN-VADILLO, O. Estabilidad primaria en implantes inmediatos versus implantes colocados en hueso maduro: un estudio clínico retrospectivo. Int. J. Odontostomat., 14(2):230-235, 2020.

hueso maduro mientras que otros autores consideran que esas diferencias sí son significativas. (West \& Oates; Rowan et al.; Han et al.; Malchiodi et al.).

El objetivo de este estudio clínico retrospectivo es comparar la estabilidad primaria de implantes inmediatos colocados en alveolos postextracción con la de implantes colocados en hueso maduro.

\section{MATERIAL Y MÉTODO}

Se llevó a cabo un estudio clínico retrospectivo de casos y controles. El tamaño total de la muestra fue de 175 implantes que se colocaron en 175 pacientes, y se dividieron en dos grupos: en el grupo A, o grupo caso, se incluyeron aquellos implantes que fueron colocados en el alveolo de forma inmediata a la extracción de un diente $(n=31)$, mientras que en el grupo $B$, o grupo control, se incluyeron los implantes colocados en hueso maduro $(n=144)$.

Criterios de inclusión: Pacientes sanos (ASA I) que necesitaran la colocación de un implante unitario para la reposición de un diente.

\section{Criterios de exclusión:}

- Controles en los que la técnica de conformación del lecho no se hiciera por fresado convencional.

- Controles en los que previamente se había realizado algún tratamiento de regeneración ósea (en altura y/o anchura).

- Pacientes con osteoporosis o defecto de densidad

- Fracasos durante el periodo de osteointegración

- Casos o controles en los que se encontró algún defecto óseo con ocupación de tejido blando.

Todos los implantes utilizados en el estudio pertenecieron al mismo modelo: Essential Cone (Klockner Implant System, Madrid, Spain) y fueron colocados por el mismo cirujano durante un año de actividad. Tanto en los casos como en los controles se siguió el protocolo de fresado recomendado por el fabricante, y en todos los implantes inmediatos se regeneró el gap vestibular con xenoinjerto particulado (Cerabone, Botiss, Berlin, Germany).

Para la medición del torque de inserción se empleó un motor de implantes con contrángulo (NSK, Tokyo, Japan), programado a dos cortes de torque de inserción: 20 y 35 Newtons ( $\mathrm{Ncm})$. En base a ello, a cada implante se le adjudicó un valor de torque en el momento de su inserción:

- 10 Ncm: aquella situación en la que para llevar el implante a su posición final en el lecho, se empleó el transportador manual que incorpora el implante.

- $15 \mathrm{Ncm}$ : aquella situación en la que para llevar el implante a su posición final en el lecho, no se consiguió de manera manual y se empleó el motor y éste no se paró al primer corte de $20 \mathrm{Ncm}$.

- $25 \mathrm{Ncm}$ : aquella situación en la que para llevar el implante a su posición final en el lecho, no se consiguió de manera manual y se empleó el motor y éste se paró al primer corte de $20 \mathrm{Ncm}$, pero no al segundo de 35 Ncm.

- $35 \mathrm{Ncm}$ : aquella situación en la que para llevar el implante a su posición final en el lecho, no se consiguió de manera manual y se empleó el motor y éste se paró al primer corte de $20 \mathrm{Ncm}$ y al segundo de $35 \mathrm{Ncm}$, teniendo que emplear la llave de carraca para acabar de colocarlo.

La medición de la estabilidad se realizó mediante un dispositivo de análisis de frecuencia de resonancia (AFR) de tercera generación Osstell ISQ (Osstell AB, Gothembourg, Sweden). Para ello, se atornilló manualmente un Smartpeg diferente para cada implante, y se midió la estabilidad en dos direcciones totalmente perpendiculares: una en sentido vestíbulo-lingual (VL) y otra en sentido mesio-distal (MD) (Fig. 1).

Los resultados obtenidos fueron analizados estadísticamente mediante un análisis estadístico descriptivo y un test Anova.

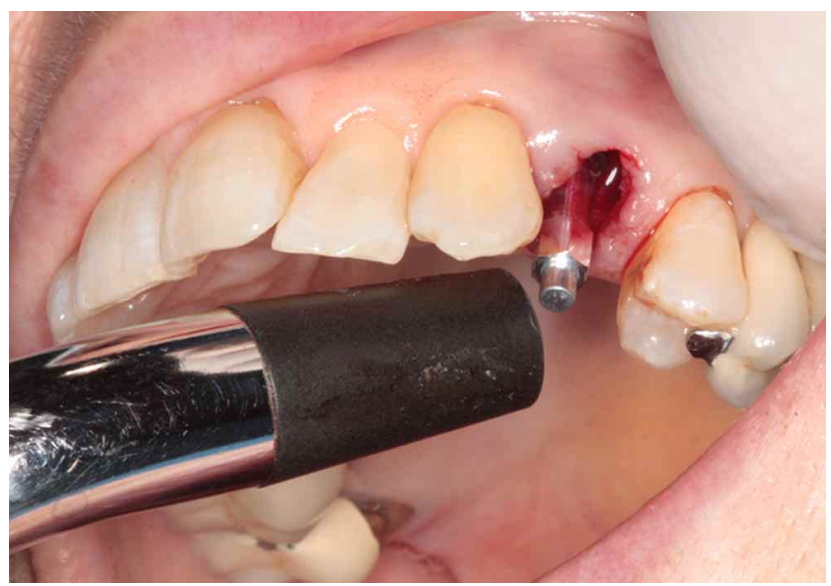

Fig. 1. Imagen clínica de la medición de estabilidad primaria mediante RFA en un implante inmediato. 
CHÁVARRI-PRADO, D.; BRIZUELA-VELASCO, A.; ORTIZ DE URBINA-COMERÓN, P.; DIÉGUEZ-PEREIRA, M.; PÉREZ-PEVIDA, E.; VITERI-AGUSTÍN, I.; ESTRADAMARTÍNEZ, A. \& MONTALBÁN-VADILLO, O. Estabilidad primaria en implantes inmediatos versus implantes colocados en hueso maduro: un estudio clínico retrospectivo. Int. J. Odontostomat., 14(2):230-235, 2020.

\section{RESULTADOS}

Los implantes inmediatos obtuvieron unos valores de torque de inserción de $26,29 \mathrm{Ncm}( \pm 10,07)$ y unos valores ISQ de 60,74 ( $\pm 6,17)$ en sentido VL y de $62,19( \pm 7,64)$ en sentido MD. Por otra parte, los resultados de los implantes colocados en hueso maduro fueron de $25,76 \mathrm{Ncm}( \pm 9,72)$ de torque, y un ISQ de $68,34( \pm 6,26)$ en sentido VL y $69,29( \pm 7,98)$ en sentido MD (Tabla I).

El análisis estadístico revela que no existen diferencias estadísticamente significativas respecto a la estabilidad medida a través del torque de inserción en $\mathrm{N} / \mathrm{cm}$ entre implantes colocados en alveolos post exodoncia e implantes colocados en hueso maduro $(p>0,05)$. Sin embargo, sí que se encontraron diferencias estadísticamente significativas en la medida de la estabilidad primaria en unidades ISQ $(p<0,05)$, siendo considerablemente inferiores los valores correspondientes a los implantes colocados en los alveolos post exodoncia, tanto en sentido VL como MD.

Además, cabe destacar que no existe correlación estadísticamente significativa entre los valores de torque de inserción y los valores ISQ.

\section{DISCUSIÓN}

En la bibliografía actual existen pocos trabajos que analicen la estabilidad primaria de los implantes inmediatos en comparación con aquellos colocados en hueso maduro. Sin embargo, podemos encontrar 4 estudios clínicos publicados que estudian este tema.

Tabla I. Resultados de comparar grupo test y grupo control.

\begin{tabular}{llcc}
\hline GRUPO & TORQUE & ISQ VL & ISQ MD \\
\hline A (grupo test) & $26,29 \pm 10,07$ & $60,74 \pm 6,17$ & $62,19 \pm 7,64$ \\
B (grupo control) & $25,76 \pm 9,72$ & $68,34 \pm 6,26$ & $69,29 \pm 7,98$ \\
\hline
\end{tabular}

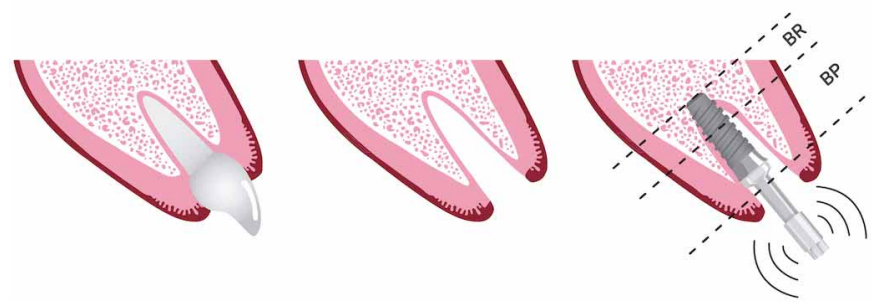

Fig. 2. Ilustración explicativa de por qué un implante inmediato puede obtener un torque de inserción alto, y al mismo tiempo un valor ISQ bajo.
Los resultados de estos trabajos muestran cierta controversia, ya que uno de ellos (Rowan et al.) demuestra la existencia de diferencias estadísticamente significativas en la estabilidad primaria de ambos grupos, mientras que los otros 3 (West \& Oates; Malchiodi et al.; Han et al.) no hallan diferencias significativas.

En el estudio de Rowan et al., un mismo cirujano colocó un total de 137 implantes en 85 pacientes, divididos en dos grupos. En el primer grupo se colocaron 41 implantes inmediatos ( 30 en el maxilar superior y 11 en la mandíbula) y 96 implantes en hueso maduro. Se tomaron cuatro mediciones del valor ISQ (vestibular, lingual, mesial y distal) y la media de estas medidas en el momento de la inserción fue de 65,60 \pm 9,33 en los implantes inmediatos y $76,73 \pm 6,5$ en los implantes colocados en hueso maduro, por lo que el análisis estadístico demostró que las diferencias fueron significativas. Esto coincide con los resultados de ISQ obtenidos en nuestro estudio. Rowan et al. no midieron el torque de inserción de los implantes, por lo que no podemos comparar sus resultados con los nuestros en cuanto a torque de inserción se refiere.

En contraposición, los resultados de nuestro estudio discrepan con los obtenidos en el trabajo de Malchiodi et al., en el que colocaron 40 implantes a 40 pacientes de los cuales 20 implantes fueron inmediatos (grupo test) y 20 implantes se colocaron en hueso maduro (grupo control). Los pacientes fueron divididos de forma aleatoria en ambos grupos. Al igual que en nuestro trabajo, los valores ISQ obtenidos en la medición de la estabilidad fueron superiores en el grupo control $(66,00 \pm 8,25)$ que en el grupo test $(61.90 \pm$ 9.99), sin embargo estas diferencias no resultaron ser estadísticamente significativas. Lo mismo ocurrió con los valores de torque $(52 \pm 9,2 \mathrm{NCm}$ en el grupo control y $46,0 \pm 9,95 \mathrm{Ncm}$ en el grupo test). Los autores del estudio concluyeron que el torque de inserción y el ISQ presentan una correlación estadística mayor en el grupo test que en el grupo control (Malchiodi et al.).

Nuestros resultados también difieren de los obtenidos por Han y colaboradores en el año 2016. Ese estudio reporta la colocación de 30 implantes inmediatos en alveolos post-extracción en 17 pacientes y 32 implantes en hueso maduro en 22 pacientes. Los valores ISQ obtenidos en el momento de la inserción fueron de $78,5 \pm 3,8$ en los implantes 
CHÁVARRI-PRADO, D.; BRIZUELA-VELASCO, A.; ORTIZ DE URBINA-COMERÓN, P.; DIÉGUEZ-PEREIRA, M.; PÉREZ-PEVIDA, E.; VITERI-AGUSTÍN, I.; ESTRADAMARTÍNEZ, A. \& MONTALBÁN-VADILLO, O. Estabilidad primaria en implantes inmediatos versus implantes colocados en hueso maduro: un estudio clínico retrospectivo. Int. J. Odontostomat., 14(2):230-235, 2020.

colocados en alveolos post extracción y $79,1 \pm 3,7$ en los colocados en hueso maduro, no habiendo diferencias estadísticamente significativas entre ambos grupos. Los autores concluyeron que tanto los implantes colocados en alveolos frescos como los colocados en hueso maduro, presentaron tasas de supervivencia y estabilidad similares, a diferencia de nuestros resultados, que demuestran valores ISQ inferiores en el grupo de los implantes inmediatos (Han et al.).

Por último, West \& Oates en el año 2007 colocaron 28 implantes inmediatos en 25 pacientes y 11 implantes en hueso maduro en 9 pacientes. La tasa de supervivencia de los implantes en sitios post-extracción de este estudio es de $96,43 \%$ y $100 \%$ en hueso maduro. El artículo muestra la inexistencia de diferencias significativas en la estabilidad primaria de los implantes de ambos grupos, medida mediante análisis de frecuencia de resonancia, aunque sí que aprecia diferencias significativas en la estabilidad secundaria a las 4, 6 y 8 semanas. Al igual que Han et al., los autores de este estudio no hacen mención al torque de inserción de los implantes, por lo que no podemos comparar dicho parámetro con los resultados de nuestro estudio (West \& Oates).

El hecho de que el torque de inserción sea similar en ambos grupos pero los valores ISQ muestren diferencias significativas puede ser debido a que ambos parámetros miden cosas diferentes. El torque hace referencia a la resistencia que encuentra un implante alrededor de su eje para avanzar girando en sentido apical, mientras que el análisis de frecuencia de resonancia proporciona mediciones objetivas sobre la rigidez de la unión hueso-implante, y sobre su micromovimiento. Este último aspecto es clave, ya que la literatura advierte que un exceso de micromovimiento se traduce en una mayor probabilidad de fracaso durante la osteointegración $(14,18)$. Estudios como el de Brizuela-Velasco et al., demuestran que no existe una correlación estadística entre ambos parámetros, y por eso pueden darse situaciones clínicas, como las de nuestro estudio, en las que los valores de torque e ISQ sean contradictorios. En el caso concreto de los implantes inmediatos, se pueden conseguir altos valores de torque durante la inserción del implante, debido al anclaje palatino y apical del mismo. Sin embargo, debido al GAP vestibular, cabe la posibilidad de que ese implante tenga un gran micromovimiento, y por lo tanto, sus valores de estabilidad primaria, determinados mediante análisis de frecuencia de resonancia sean bajos (Fig. 2).
Desde un punto de vista clínico, esto tiene una gran relevancia, ya que puede condicionar enormemente el pronóstico de nuestros tratamientos. En el caso concreto de los implantes inmediatos de los que habla nuestro estudio, a menudo se da la situación de que el clínico está valorando la posibilidad de realizar una carga inmediata sobre el implante inmediato recién colocado. Si para la toma de esta decisión se basa exclusivamente en los valores de torque, este le puede jugar una mala pasada aportándole falsos positivos. Es decir, el torque expresa un grado alto de estabilidad, adecuado para realizar carga inmediata, y sin embrago el implante presenta un grado alto de micromovimiento, por lo que presenta un alto riesgo de fracaso durante la osteointegración.

Dados los resultados obtenidos en este estudio, podemos obtener las siguientes conclusiones:

La estabilidad primaria de los implantes inmediatos es similar a la de los implantes colocados en hueso maduro cuando la medimos mediante el torque de inserción.

Sin embargo, el análisis de frecuencia de resonancia demuestra que en realidad, la estabilidad de los implantes inmediatos es significativamente inferior que en los implantes diferidos, debido a su mayor grado de micromovimiento en base al GAP vestibular resultante entre el hueso y el implante.

En la toma de decisiones sobre la conveniencia de adoptar protocolos de carga inmediata o temprana, es de vital importancia conocer los valores ISQ del implante, ya que el torque de inserción puede proporcionar falsos positivos.

CHÁVARRI-PRADO, D.; BRIZUELA-VELASCO, A.; ORTIZ DE URBINA-COMERÓN, P.; DIÉGUEZ-PEREIRA, M.; PÉREZ-PEVIDA, E.; VITERI-AGUSTÍN, I.; ESTRADAMARTÍNEZ, A. \& MONTALBÁN-VADILLO, O. Primary stability in immediate implants Vs implants placed in mature bone: A retrospective clinical study. Int. J. Odontostomat., 14(2):230235, 2020.

ABSTRACT: Primary stability is an important requirement for the survival and success of implants during osseointegration. In recent years, immediate post-extraction implants have proven to be a successful and predictable treatment option for the replacement of teeth with a poor prognosis, but the question arises as to whether these implants reach primary stability values comparable to those placed in mature bone. The objective of the study was to compare the primary stability of immediate implants placed in post-extraction 
CHÁVARRI-PRADO, D.; BRIZUELA-VELASCO, A.; ORTIZ DE URBINA-COMERÓN, P.; DIÉGUEZ-PEREIRA, M.; PÉREZ-PEVIDA, E.; VITERI-AGUSTÍN, I.; ESTRADAMARTÍNEZ, A. \& MONTALBÁN-VADILLO, O. Estabilidad primaria en implantes inmediatos versus implantes colocados en hueso maduro: un estudio clínico retrospectivo. Int. J. Odontostomat., 14(2):230-235, 2020

alveoli with that of implants placed in mature bone. A retrospective clinical study was carried out, with data collected on 175 implants, placed in 175 patients. All implants placed belonged to the Essential Cone model (Klockner Implant System) and were divided into two groups: immediate implants (Group A, $n=31$ ) and implants placed in mature bone (Group B, $n=144)$. The primary stability of all implants was measured by insertion torque and resonance frequency analysis with Osstell ISQ. No statistically significant differences were found regarding the stability measured through the insertion torque $(26.29+10.07$ Vs $25.76+9.72 \mathrm{~N} / \mathrm{cm}$ ) but significant differences were found in the measurement of primary stability by means of AFR, the values corresponding to implants placed in the post-exodontic alveoli being lower $(60.74 \pm 6.17$ in the VL direction and $62.19 \pm 7.64$ in the MD direction versus $68.34 \pm 6.26$ in the $\mathrm{VL}$ direction and $69.29 \pm 7.98$ in the MD direction obtained in implants placed in mature bone). The insertion torque of immediate implants is similar to that of implants placed in mature bone, but their ISQ values are significantly lower, which demonstrates a higher degree of micromotion, and therefore, a greater risk of failure during the period of osseointegration.

KEY WORDS: implant stability, primary stability, resonance frequency analysis, insertion torque, immediate implant.

\section{REFERENCIAS BIBLIOGRÁFICAS}

Adell, R.; Lekholm, U.; Rockler, B. \& Brånemark, P. I. A 15-year study of osseointegrated implants in the treatment of the edentulous jaw. Int. J. Oral Surg., 10(6):387-416, 1981.

Agliardi, E.; Panigatti, S.; Clericò, M.; Villa, C. \& Malò, P. Immediate rehabilitation of the edentulous jaws with full fixed prostheses supported by four implants: interim results of a single cohort prospective study. Clin. Oral Implants Res., 21(5):459-65, 2010.

Bilhan, H.; Geckili, O.; Mumcu, E.; Bozdag, E.; Sünbüloglu, E. \& Kutay, $\mathrm{O}$. Influence of surgical technique, implant shape and diameter on the primary stability in cancellous bone. J. Oral Rehabil., 37(12):900-7, 2010.

Brånemark, P. I. Tooth replacement by oral endoprostheses: clinical aspects. J. Dent. Educ., 52(12):821-3, 1988.

Brizuela-Velasco, A.; Álvarez-Arenal, Á.; Gil-Mur, F. J.; HerreroCliment, M.; Chávarri-Prado, D.; Chento-Valiente, Y. \& DieguezPereira, M. Relationship between insertion torque and resonance frequency measurements, performed by resonance frequency analysis, in micromobility of dental implants: an in vitro study. Implant Dent., 24(5):607-11, 2015.

Chen, S. T.; Wilson Jr., T. G. \& Hämmerle, C. H. F. Immediate or early placement of implants following tooth extraction: review of biologic basis, clinical procedures, and outcomes. Int. J. Oral Maxillofac. Implants., 19 Suppl.:12-25, 2004

Dos Santos, M. V.; Elias, C. N. \& Cavalcanti Lima, J. H. The effects of superficial roughness and design on the primary stability of dental implants. Clin. Implant Dent. Relat. Res., 13(3):215-23, 2011.

Gehrke, S. A. \& Marin, G. W. Biomechanical evaluation of dental implants with three different designs: removal torque and resonance frequency analysis in rabbits. Ann. Anat., 199:30-5, 2015.

Han, C. H.; Mangano, F.; Mortellaro, C. \& Park, K. B. Immediate loading of tapered implants placed in postextraction sockets and healed sites. J. Craniofac. Surg., 27(5):1220-7, 2016.
Koh, J. W.; Yang, J. H.; Han, J. S.; Lee, J. B. \& Kim, S. H. Biomechanical evaluation of dental implants with different surfaces: Removal torque and resonance frequency analysis in rabbits. J. Adv. Prosthodont., 1(2):107-12, 2009.

Lazzara, R. J. Immediate implant placement into extraction sites: surgical and restorative advantages. Int. J. Periodontics Restorative Dent., 9(5):332-43, 1989.

Lin, C. L.; Chang, S. H.; Chang, W. J. \& Kuo, Y. C. Factorial analysis of variables influencing mechanical characteristics of a single tooth implant placed in the maxilla using finite element analysis and the statistics-based taguchi method. Eur. J. Oral Sci., 115(5):408-16, 2007.

Lindeboom, J. A.; Tjiook, Y. \& Kroon, F. H. M. Immediate placement of implants in periapical infected sites: a prospective randomized study in 50 patients. Oral Surg. Oral Med. Oral Pathol. Oral Radiol. Endod., 101(6):705-10, 2006.

Malchiodi, L.; Balzani, L.; Cucchi, A.; Ghensi, P. \& Nocini, P. F. Primary and secondary stability of implants in postextraction and healed sites: a randomized controlled clinical trial. Int. J. Oral Maxillofac. Implants, 31(6):1435-43, 2016.

McCullough, J. J. \& Klokkevold, P. R. The effect of implant macro-thread design on implant stability in the early post-operative period: a randomized, controlled pilot study. Clin. Oral Implants Res., 28(10):1218-26, 2017.

Misch, C. E.; Suzuki, J. B.; Misch-Dietsh, F. M. \& Bidez, M. W. A positive correlation between occlusal trauma and peri-implant bone loss: literature support. Implant Dent., 14(2):108-16, 2005.

Pjetursson, B. E.; Tan, K.; Lang, N. P.; Brägger, U.; Egger, M. \& Zwahlen, M. A systematic review of the survival and complication rates of fixed partial Dentures (FPDs) after an observation period of at least 5 years. Clin. Oral Implants Res., 15(6):625-42, 2004.

Rowan, M.; Lee, D.; Pi-Anfruns, J.; Shiffler, P.; Aghaloo, T. \& Moy, P. K. Mechanical versus biological stability of immediate and delayed implant placement using resonance frequency analysis. J. Oral Maxillofac. Surg., 73(2):253-7, 2015.

Santamaría-Arrieta, G.; Brizuela-Velasco, A.; Fernández-González, F. J.; Chávarri-Prado, D.; Chento-Valiente, Y.; Solaberrieta, E.; DiéguezPereira, M.; Vega, J. A. \& Yurrebaso-Asúa, J. Biomechanical evaluation of oversized drilling technique on primary implant stability measured by insertion torque and resonance frequency analysis. $J$. Clin. Exp. Dent., 8(3):e307-11, 2016.

Sennerby, L. Resonance frequency analysis for implant stability measurements. A review. Integr. Diagn. Update, 1:1-11, 2015.

Werbitt, M. J. \& Goldberg, P. V. The immediate implant: bone preservation and bone regeneration. Int. J. Periodontics Restorative Dent., 12(3):206-17, 1992.

West, J. D. \& Oates, T. W. Identification of stability changes for immediately placed dental implants. Int. J. Oral Maxillofac. Implants, 22(4):62330, 2007.

Dirección para correspondencia:

David Chávarri Prado

Especialista Universitario en Implantología Oral (UPV-EHU)

Máster en Investigación Biomédica (UPV-EHU)

Clínica Dental Reyes de Navarra

Vitoria-Gasteiz

ESPAÑA

Email: davidchavarri@hotmail.com

Recibido: 27-09-2019

Aceptado:16-12-2019 